\title{
WSN based Cost Effective Intelligent Traffic Light Control System based on Image Processing
}

\author{
Anuradha G. Suratekar \\ Mtech Student \\ Department of Technology \\ Shivaji University, Kolhapur
}

\author{
Uttam L. Bombale \\ Associate Professor \\ Department of Technology \\ Shivaji University, Kolhapur
}

\begin{abstract}
In this paper image processing based intelligent traffic light control system is designed. Inductive loop method is mostly used for traffic detection. But it is physically large, hard to install and maintain. Present system has fixed timing for traffic control so the present traffic system is not much efficient to manage or control the traffic. Also inductive loops cannot communicate with each other. The wireless sensor network (WSN) has features like fault tolerance, scalability, real-time, and coordination. So the traffic light control system as per the traffic density with WSN has been designed in this paper. $\mathrm{C}++$ programming language is used for simulation.
\end{abstract}

\section{Keywords}

WSN, image processing, $\mathrm{C}++$ programming language

\section{INTRODUCTION}

Major cities are suffering by traffic congestion problem. Present fixed time traffic control system is insufficient to control the traffic flow and causes traffic jam problems. These traffic congestion problems are having a deep impact on companies production which has lead to economic crises in the country. Most common detection systems to measure traffic flow on roads are inductive loops and infrared object sensors [1-3], but these are unable to communicate with each other. For communication LAN and RFID based systems are better solution $[4,5]$ which is costly to deploy and maintain. Massachusetts Institute of Technology Electrical Science and Engineering had also built a wireless sensor network for Smart Roadbeds traffic control using magnetic sensors to replace inductive loop to monitor the traffic flow rate during free-flowing traffic and a traffic jam for a single intersection [6]. The German Aerospace Center DLR Institute for Transport Research used number of camera nodes to replace with the inductive loop to capture a traffic objects, then using real-time algorithms for image processing and a new approach for dynamic traffic light management for a single intersection traffic control [7]. The motivation behind the implementation of wireless sensor network (WSN) along with image processing is cost effective technology that bear a pivotal potential to overcome these difficulties [8]. A Wireless Sensor Network allows the integration of video monitoring with magnetic or power sensor to obtain complete and integrated information like video-images and traffic volumes information [9]. This technology enables a new broad range of smart city applications around urban sensing including traffic safety, traffic congestion control, road state monitoring, vehicular warning services, and parking management. In [10], authors survey vehicular sensor network (VSN) platforms, while those of [11] discuss many urban applications.

\section{PROPOSED SYSTEM}

In existing system green light will be on for fixed time duration even there is heavy traffic on a single road as compare to other roads i.e. existing system does not understand the actual traffic condition which causes congestion problem, leads to economic and environmental crises. Also there is traffic control system based on image processing using Matlab. But this system is costly. To overcome these problems the traffic control system has been developed which will control the traffic at one intersection and also communicate with other intersection using Wireless Sensor Network (WSN). In Figure 1 we described the proposed system. This system consists of three units, as described below.

\subsection{Sensing Unit}

In sensing unit camera is acting as a sensing element. Camera is mounted on a top of the signal. It captures the image of traffic loaded roads simultaneously. Vehicle count will be generated using image processing method. For image processing we are using programming language like $\mathrm{C}++$ with OpenCV libraries. This vehicle count is further given to the controlling unit serially.

\subsection{Controlling Unit}

In controlling unit count will be accepted by sensing unit using serial communication. Then serial to parallel data conversion will take place at the microcontroller part. After comparing these four counts the road which has maximum density will get access first. At the same time comparison between other three roads will be calculated continuously. When density of that road becomes less or time allotted for it gets over then access will be given to the road which has maximum density in between these three roads. At the same time this information is shared with other intersection using Zigbee modules.

\subsection{Communication Unit}

For communication between two intersections we are using Zigbee module which is a low-cost, low-power, wireless mesh networking standard. Zigbee employs a suite of technologies to enable scalable, self-organizing, self-healing networks that can manage various data traffic patterns. Zigbee is used for communication between other intersections about vehicle count so that at other intersection controller can control green signal according to present vehicles on that intersection and the vehicles coming from other intersections. 


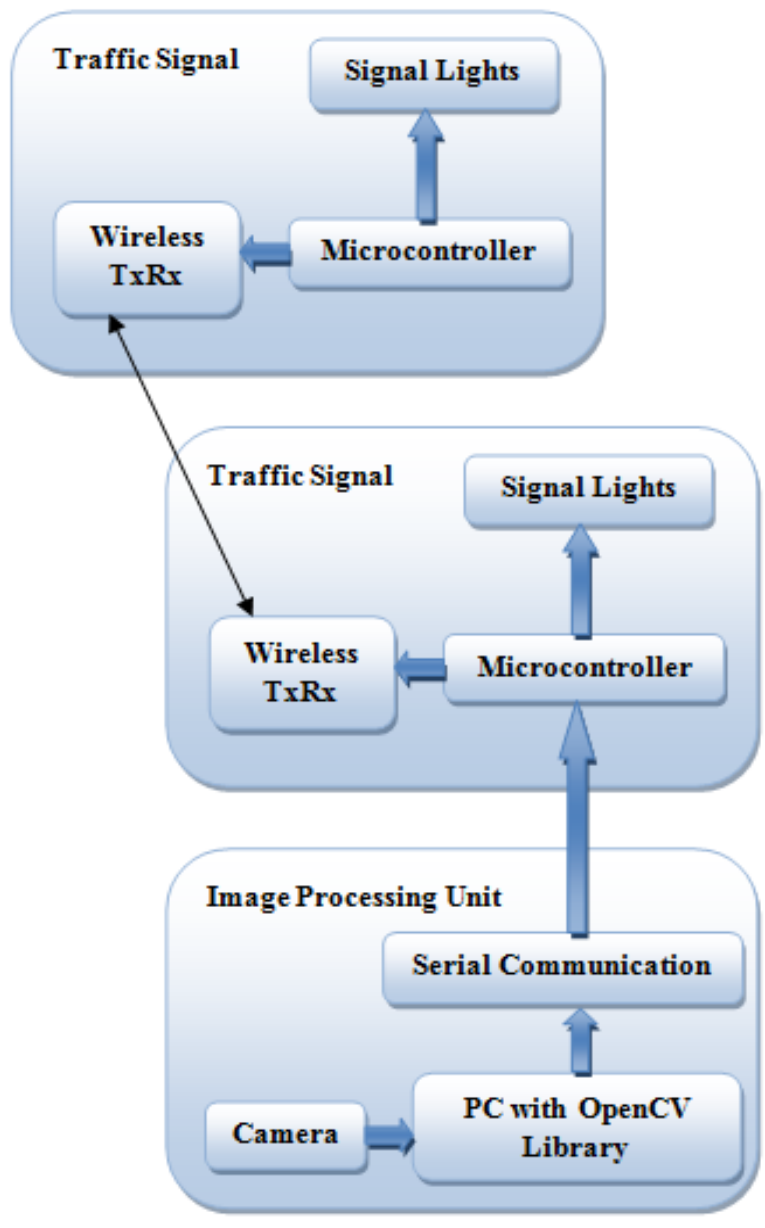

Figure 1: Block Diagram of Proposed System

\section{WORKING PRINCIPLE}

\subsection{Algorithm}

1. Initialize camera.

2. Get image from camera. And convert it into RGB.

3. Convert RGB image into HSV. Separate hue plane.

4. Separate out four roads.

5. Apply background separation.

6. Apply morphological operation on it to calculate objects.

7. Check the count of vehicles using $\mathrm{C}++$ programming language.

8. Fed it to microcontroller serially.

9. As per the count, control traffic lights.

\subsection{Related Work}

\subsubsection{Canny Edge Detection}

The Canny edge detection technique, mostly used now-a-days is developed by John Canny [12] in 1986. The Canny edge detection algorithm had three main objectives [13]: 1. Optimal edge detection with no false positives 2. Good localization with minimal distance between the detected and actual edge position 3. Eliminate multiple segments and instead create a single longer edge. In canny edge detection to eliminate false edges it uses Gaussian smoothing. In next step sobel operator is used to produce both magnitude and direction information for the edges. Hysteresis thresholding is used for the pixels found in the non-maximum suppression step and create an edge segment by exploring the neighboring pixels from the peaks.

\subsubsection{Dilation}

Dilation is used to expand the detected edges using structuring element. There are three types of structuring elements [14]: 1 . MORRPH_RECT - It is a rectangular structuring element with Eij=1. 2. MORPH_ELLIPSE - It is an elliptic structuring element, that is, a filled ellipse inscribed into the rectangle $\operatorname{Rect}(0,0$, esize.width, 0.esize.height) 3. MORPH_CROSS It is a cross-shaped structuring element. Eij $=1$ if $\bar{i}=$ anchor.y (y-coordinate of the anchor) or $\mathrm{j}=$ anchor. $\mathrm{x}$ ( $\mathrm{x}$-coordinate of the anchor) and $\mathrm{Eij}=0$ otherwise. Anchor position is within the element. The default value is $(-1 ;-1)$ i.e. the anchor is at the center. Only the shape of a cross-shaped element depends on the anchor position. 4. CV_SHAPE_CUSTOM - It is a custom structuring element (OpenCV 1.x API).

\subsubsection{Hole Filling}

After detecting the contour this contour is filled by white color and that will be detect as a single object. After hole filling small objects will be removed and the object will be count for further operations.

\section{RESULT AND DISCUSSION}

A complete prototype for intelligent traffic control system is developed. The system has been tested with different conditions of vehicles on each road. This system has four roads namely road1, road2, road3, road4 as shown in Figure 2.

\begin{tabular}{|l|l|l|}
\hline & Road 1 & \\
\hline Road 2 & & \\
\hline & & Road 4 \\
\hline & Road 3 & \\
\hline
\end{tabular}

Figure 2: Four Roads at an Intersection for Comparison

Here reference image without any vehicles and current image with different number of vehicles get compared to detect count of vehicles.

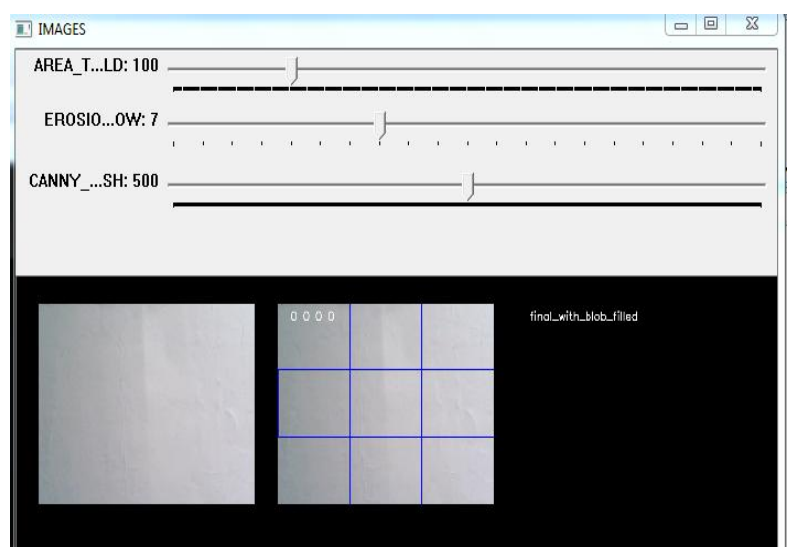

Figure 3: Reference Image 
For reference image count for all the roads are zero. When vehicles start coming at the junction count goes on changing on each road as shown in Figure 4.

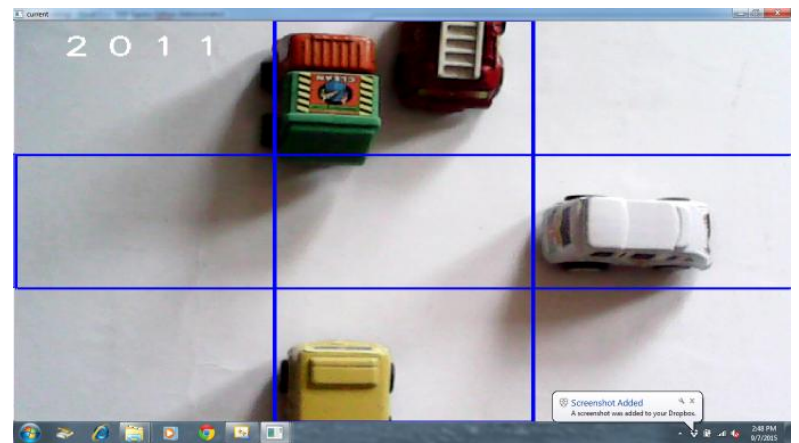

Figure 4: roads with different number of vehicles

By comparing reference image of road and current image of road to detect count, difference between these two images is taken simultaneously.

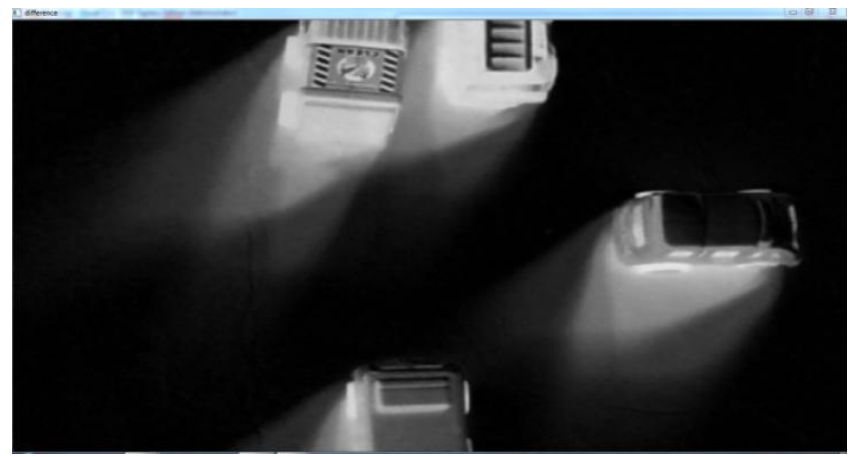

Figure 5: Output of Difference between Reference Image and Current Image

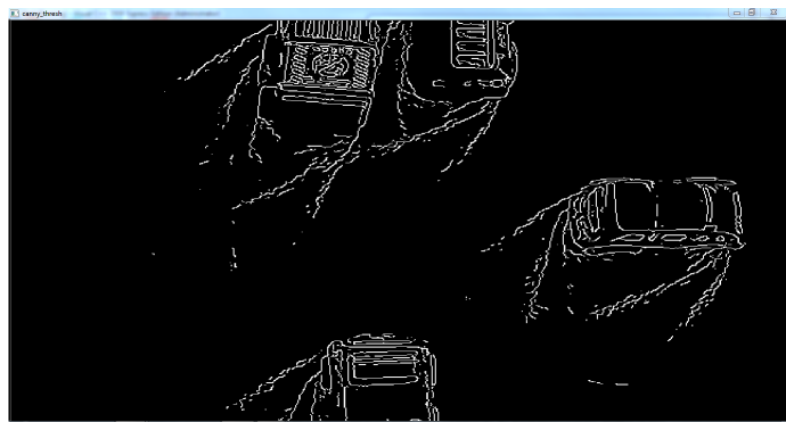

Figure 6: Output of Canny Edge Detection

Using canny edge detection operator we can detect closed loop in the image as shown in Figure 6. After detecting edges, using blob filling operation we removed background and consider only foreground objects for comparison.

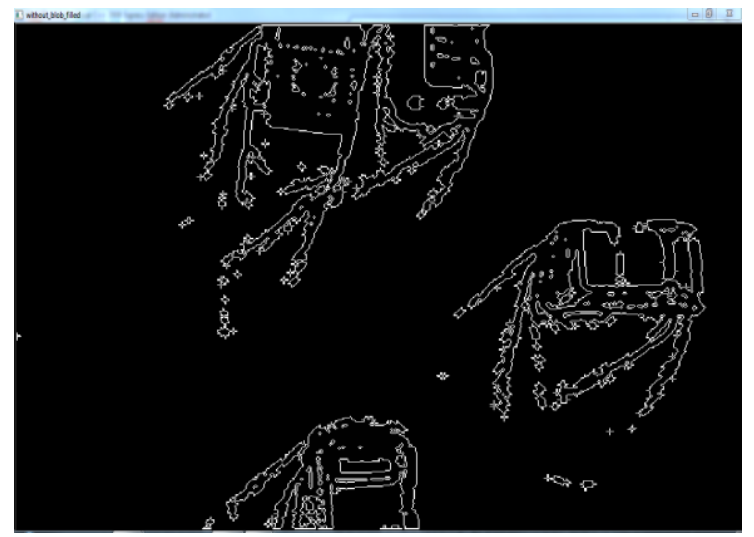

Figure 7: Output without Blob Filling Operation

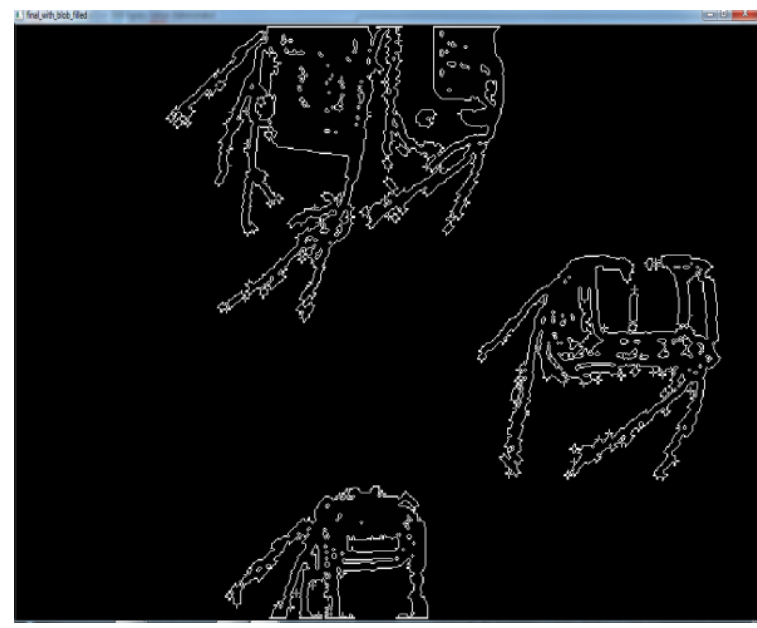

Figure 8: Output with Blob Filling Operation

\section{CONCLUSION}

This paper discusses the WSN based traffic control system using single camera for capturing at the intersection and provides input image to the image processing unit. As OpenCV is an open source library system becomes cost effective. In future to detect the traffic density more than one camera can be used at each intersection. Using one camera for each road at intersection system can get perfect count for each road. Also for emergency exits like ambulance, fire bridged, we can use GPS in such vehicles for continuous tracking of that vehicle.

\section{REFERENCES}

[1] L. A. Klein, M. R. Kelley, M. K. Mills, "Evaluation of overhead and in-ground vehicle detector technologies for traffic flow measurement," Journal of Testing and Evaluation, 25(2): pp.205-214, 1997.

[2] K. Tavladakis, N. C. Voulgaris, "Development of an autonomous adative traffic control system," in Proc. European Symposium on Intelligent Techniques, 1999.

[3] A. Albagul, M. Hrairi, Wahyudi, M. F. Hidayathullah, "Design and development of sensor based traffic light system," American Journal of Applied Sciences, 3(3): pp.1745-1749, 2006.

[4] Shwe Yi Aye, "Design and construction of LAN based car traffic control system," in Proc. WASET, 2008. 
[5] Khalid A. S. Al-Khateeb, Jaiz A. Y. Johari, Wajdi F. AlKhateeb, "Dynamic traffic light sequence algorithm using RFID," Journal of Computer Science, 4(7): pp.517-524, 2008.

[6] Li JianZhong, Li JinBao, Shi ShengFei, "Concepts, Issues and Advance of Sensor Networks and Data Management of Sensor Networks," Journal of Software, vol. 14, no. 10, pp. 1717-1727, 2003

[7] D. Abbott, S. Cunningham, G. Daniels, B. Doyle, J. Dumlop, D. Economo, T. Farmer, D. Farrant, C. Foley, B. Fox, M. Hedley, J. Herrmann, C. Jacka, "Development and Evaluation of Sensor Concepts for Ageless Aerospace Vehicles - Threats and Measurands," NASA, vo. 2, no. 11, pp. 68-77, 2002.

[8] S. Deshpand, "Adaptative low-bitrate streaming over IEEE 802.15.4 low rate wireless personal area networks (LR-WPAN) based on link quality indication", International Conference on Communication and Mobile Computing, pp. 863-868, 2006.
[9] G. Pekhterverv et. al "Image Transmission over IEEE 802.15.4 and ZigBee Networks" IEEE International Symposium on Circuits and Systems, Vol.4, pp.35393543,2005

[10] Uichin Lee, Mario Gerla. "A survey of urban vehicular sensing platforms" Computer Networks 54 (2010) 527 544.

[11] Abhijit Sharma, Rituparna Chaki, Uma Bhattacharya. "Applications of WSN in ITS: A Review". ICECT11.

[12] CANNY, J. A computational approach to edge detection. IEEE Transactions on Pattern Analysis and Machine Intelligence 8, 6 (1986), 679-686.

[13] NIXON, M. S., AND AGUADO, A. S. Feature Extraction and Image Processing, second ed. Elsevier, New York, 2008.

[14] The OpenCV Reference Manual. 